\title{
Multi-Population Differential Evolution for Retinal Blood Vessel Segmentation
}

\author{
Kamlesh Mistry, Biju Issac, Seibu Mary Jacob, Jyoti Jasekar and Li Zhang
}

\begin{abstract}
The retinal blood vessel segmentation plays a significant role in the automatic or computer-assisted diagnosis of retinopathy. Manual blood vessel segmentation is very timeconsuming and requires a great amount of domain knowledge. In addition, the blood vessels are only a few pixels wide and cover the entire fundus image. This further hinders the recent systems from automating the retinal blood vessel segmentation efficiently. In this paper, we propose a modified differential evolution (DE) algorithm to carry out automatic retinal blood vessel segmentation. The modified DE employs cross-communication among multiple populations to select three types of features i.e. thick blood vessels, thin blood vessels and non-blood vessels. Multiple classifiers such as neural networks (NN), Support vector machines (SVM), NN based and SVM based ensembles are used to further measure the performance of segmentation. The proposed algorithm is evaluated on three publicly available retinal image datasets like DRIVE, STARE and HRF. It outperformed the state-of-the-art with a high average accuracy of $98.5 \%$ along with high sensitivity and specificity.
\end{abstract}

\section{INTRODUCTION}

According to the World Health Organization, around 285 million people in the world were visually impaired in 2010 and they have found that $80 \%$ of the visual impairment cases are treatable or can be prevented [1]. The changes in retinal blood vessels provide very important information on the medical diagnosis and possible directions on further treatments. Retinal images that are done by a highly specialized camera called fundus camera helps in diagnosing several eye diseases such as diabetic retinopathy, glaucoma, retinal artery occlusion, branch vein occlusion, central retinal vein occlusion, diabetes etc. Besides the retinal diseases, the diseases such as stroke, hypertension and diabetes also produce noticeable alterations in the retinal blood vasculature [2]. Examples of healthy and unhealthy retinal images are shown in Figure 1 and since the blood vessels are generally quite thin, accurate segmentation is a difficult task. The search and optimization are a classical problem that needs to be addressed. Given the constraints and flexibilities, optimization techniques will find the best solution for a given problem. In evolutionary computation, differential evolution (DE) is developed by Rainer Storn and Kenneth Price [3] and is a

Kamlesh Mistry is with Department of Computer and Information Sciences, Northumbria University, UK k.mistry@northumbria.ac.uk

Biju Issac is with Department of Computer and Information Sciences, Northumbria University, UK bis saca i eee. org

Seibu Mary Jacob is with School of Engineering, Science and Design, Teesside University, UK s. jacobetees. ac.uk

Jyoti Jasekar is with Northumbria Healthcare, NHS Stokesley, UK jyoti.jasekaregmail. com

Li Zhang is with Department of Computer and Information Sciences, Northumbria University, UK li.zhang@northumbria.ac.uk

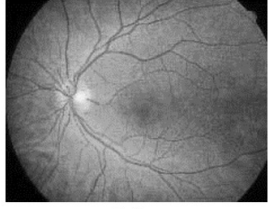

(a)

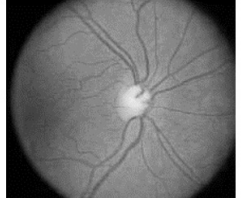

(b)

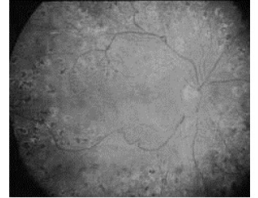

(c)
Fig. 1. (a) Normal Fundus (b) Glaucoma and (c) Diabetic Retinopathy

method that uses iteration to optimize a problem by improving a candidate solution with respect to a measure of quality. DE is a simple real parameter optimization algorithm and it works through a simple cycle of stages, presented in Figure 2 as follows: initialization of vectors, difference vector-based mutation, crossover or recombination and selection [4]. We will explain these stages further in the sections below.

This paper explores the discrimination capabilities in the texture of fundus to differentiate between healthy and unhealthy images, where the focus is to examine the performance of an extended variant of differential evolution (DE) to carry out automatic retinal blood vessel segmentation. The segmentation of blood vessels in the fundus image and further analysis of its properties aids in the diagnosis of retinal vascular disorders, as changes in the vascular network, produce changes to its shape, width, and branching pattern. Hence extraction of vascular points is useful for image registration [5]. The segmentation of blood vessels is a timeconsuming process, even for the experts. The utilization of computational intelligence to automatically segment the blood vessels in retinal images through a portable fundus camera and classify it with high accuracy would be useful for various medical personnel, which is the motivation for this work.

This paper is organized as follows. The related works is discussed in section 2. Differential evolution (DE) is explained in section 3 and the proposed system with the modified version of $\mathrm{DE}$ along with classifiers is explained in section 4 . The evaluation is done in section 5 by comparing the performance of the proposed system with the state of the art and also by discussing the convergence. The conclusion is in section 6 .

\section{RELATED WORKS}

The authors in [6] proposed an automated retinal blood vessel segmentation based on artificial bee colony optimization in conjunction with fuzzy c-means clustering. To find cluster centres of the fuzzy c-means objective function, artificial bee colony optimization is used as a global search method. To localize small vessels with a different fitness function, they 


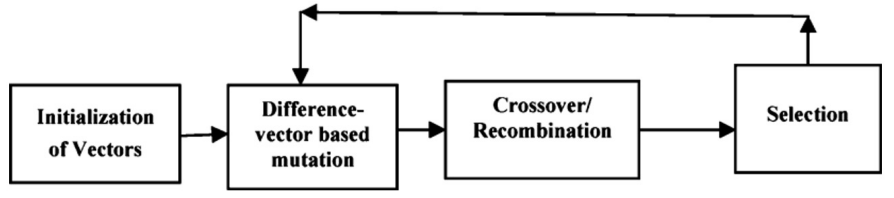

Fig. 2. Main stages of the DE algorithm [Das and Suganthan, 2011]

used a pattern search approach to optimization. Hassan et al. [7] presented a blood vessel segmentation approach for extracting the vasculature on retinal fundus images based on the Particle Swarm Optimization (PSO) to determine the n-1 optimal n-level thresholds on retinal fundus images. The idea was tested on the DRIVE datasets and its efficiency was compared with alternative methods. Correlation clustering, a graph-partitioning algorithm often used in natural language processing and document clustering, has been made better by improving the basic correlation clustering formulation by taking into account higher-order cluster relationships, thus making it effective for image segmentation algorithms [8].

The proposed approach by Sreejini and Govindan [9] makes use of the improved noise suppression features of multiscale Gaussian matched filter with the right parameter values of the filter through particle swarm optimization for achieving improved accuracy of retinal vessel segmentation. Arnay et al. [10] presented the optic cup segmentation in retinal fundus images through an Ant Colony Optimization approach. The artificial agents will produce solutions through a heuristic that uses the in-tensity gradient of the optic disc area and the curvature of the vessels. The exploration capabilities of the agents are limited on its own, but by sharing the experience of the en-tire colony, they could obtain accurate cup segmentations, even in images with a weak or nonobvious pallor. In this paper by Wang et al. [11], a supervised method that combines two superior classifiers Convolutional Neural Network (CNN) and Random Forest (RF) is presented to deal with retinal blood vessel segmentation.

\section{DifFERENTIAL EVOLUTION}

The four stages of Differential Evolution (DE) algorithm are explained below [4]. In order to improve the overall performance of the system under a given set of conditions, we need to optimize the system parameter values which are real numbers for the best performance. The system performance parameters can be represented as a vector $\vec{X}=\left[x_{1}, x_{2}, \cdots, x_{D}\right]^{T}$ where $x_{i}$ is a real number. A fitness or objective function is designed to measure the performance. The optimization task is to find a parameter vector $\vec{X} *$ which minimizes the objective function $f(\vec{X})\left(f: \Omega \subseteq R^{D} \rightarrow R\right)$ where $f(\vec{X} *)<f(\vec{X})$ for all $\vec{X} \in \Omega$ where $\Omega$ is a large finite set that is non-empty which forms the search domain. $R^{D}$ is a D-dimensional real parameter space. An optimization task can be complicated by the existence of non-linear objective functions with multiple local minima. A local minimum $f_{l}=f\left(\vec{X}_{l}\right)$ is given as follows:

$$
\exists \epsilon>0, \forall \vec{X} \epsilon \Omega:\left\|\vec{X}-\vec{X}_{l}\right\|<\epsilon \Rightarrow f_{l} \leq f(\vec{X})
$$

where $\|$.$\| shows the p-norm distance measure.$

\section{A. Initialization of vectors}

DE looks for a global optimum point in $R^{D}$, the real parameter space starting with a random population of NP D dimensional real-valued parameter vectors that could represent genome or chromosome. The different generations of DE are denoted by $G=0,1, \ldots G_{\max }$. The $i^{t} h$ vector of the population at the current generation can be shown as follows: $\vec{X}_{i, G}=\left[x_{1, i, G}, x_{2, i, G}, x_{3, i, G}, \ldots, x_{D, i, G}\right]$. For the initial population of at $\mathrm{G}=0$, with each parameter value restricted within a certain range, the prescribed maximum and minimum bounds are as follows, $\vec{X}_{\text {min }}=\left[x_{1, \min }, x_{2, \min }, \ldots, x_{D, \min }\right]$ and $\vec{X}_{\max }=\left[x_{1, \max }, x_{2, \max }, \ldots, x_{D, \max }\right]$. We may initialize the $j^{\text {th }}$ component of the $i^{\text {th }}$ vector as:

$$
x_{j, i, 0}=x_{j, \min }+\operatorname{rand}_{i, j}[0,1] .\left(x_{j, \max }-x_{j, \min }\right)
$$

\section{B. Mutation with Difference vectors}

Mutation is the change that happens with a random element. The parent vector or target vector from the current generation when combined with the mutant vector or donor vector produced through differential mutation produces an offspring called trail vector. To create a donor vector for each $i^{t h}$ target from the current population, three different parameter vectors $\vec{X}_{r_{1}^{i}}, \vec{X}_{r_{2}^{i}}, \vec{X}_{r_{3}^{i}}$, where the three $r_{x=1,2 \text { or } 3}^{i}$ indices are mutually exclusive and random integers from the range [1, $\mathrm{NP}$, which is different from the base vector index $i$. The difference of any two of these three vectors is multiplied by a scalar $\mathrm{F}$, where $0.4<\mathrm{F}<1$ and the scaled difference is added to the third vector to obtain the donor vector as follows:

$$
\vec{V}_{i, G}=\vec{X}_{r_{1}^{i}}+F \cdot\left(\vec{X}_{r_{2}^{i}}-\vec{X}_{r_{3}^{i}}\right)
$$

\section{Crossover}

To increase the population diversity, a crossover is done where the donor vector exchanges its components with the target vector $\vec{X}_{i, G}$ to form the trail vector which can be represented as $\vec{U}_{i, G}=\left[\vec{U}_{1, i, G}, \vec{U}_{2, i, G}, \vec{U}_{3, i, G} \ldots, \vec{U}_{D, i, G}\right]$. The crossover can be two types, exponential and binomial. In exponential crossover, a random integer $\mathrm{n}$ is chosen such that $1<\mathrm{n}<\mathrm{D}$. With this starting point in the target vector another integer $\mathrm{L}$ is chosen such that $1<\mathrm{L}<\mathrm{D}$ which denotes the number of components the donor vector contributes to the target vector. The trail vector is obtained as follows:

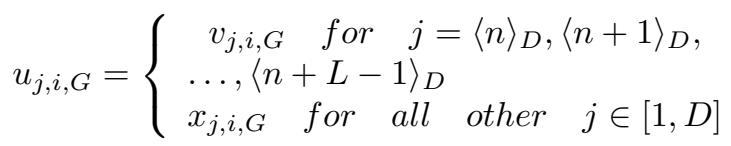

where the angular brackets show the modulo function with modulus $\mathrm{D}$. The integer $\mathrm{L}$ is from $[1, \mathrm{D}]$. The crossover $C_{r}$ rate can be seen to satisfy the condition $(L=V)=$ $\left(C_{r}\right) v-1$ for any positive integer $\mathrm{v}$ in the interval [1,D]. But the binomial crossover is done on each of the $\mathrm{D}$ variables when the randomly generated number between 0 and 1 is less than or equal to Cr. The process can be shown as follows:

$$
u_{j, i, G}= \begin{cases}v_{j, i, G} & \text { if }\left(\operatorname{rand}_{i, j}[0,1] \leq C_{r} \quad \text { or } \quad j=j_{\text {rand }}\right) \\ x_{j, i, G} & \text { otherwise }\end{cases}
$$


where $\operatorname{rand}_{i, j}[0,1]$ is a uniformly distributed random number which is called anew for each $j^{\text {th }}$ component of the $i^{\text {th }}$ parameter vector and $j_{\text {rand }} \epsilon[1,2, \ldots D]$ is a random index.

\section{Selection}

To have a constant population size over different generations, the selection is used to determine the target or trail vector survives to the next generation, which is at $\mathrm{G}=\mathrm{G}+$ 1. The selection can be shown as follows:

$$
\vec{X}_{i, G+1}= \begin{cases}\vec{U}_{i, G} & \text { if } f\left(\vec{U}_{i, G}\right) \leq f\left(\vec{X}_{i, G}\right) \\ \vec{X}_{i, G} & \text { if } f\left(\vec{U}_{i, G}\right)>f\left(\vec{X}_{i, G}\right)\end{cases}
$$

where $f(\vec{X})$ is the objective function to be minimized.

\section{The Proposed System - A Modified DE with CLASSIFIERS}

The conventional Differential Evolution only employs one set of the population to explore the search space. The single set of population restricts the search in only one direction or one set of features. The application of retinal blood vessel segmentation can lead to multiple clusters of features, which makes conventional DE less useful in this scenario.

In this paper, we propose a modified DE which employs multiple populations in order to cluster the features. This work is novel as no one has attempted a differential evolution variant on retinal images. The proposed DE starts by randomly initializing the $\mathrm{k}$ number of populations. The same number of population size $N_{p}$ is assigned to all populations. The fitness evaluation of each population is conducted using a separate criterion where the populations will look for thick blood vessels pixels, thin blood vessels pixels and non-blood vessel pixels. Now, after mutation and crossover each population will be able to generate a set of multiple features. However, this approach leads to running multiple DE in parallel independently. This means the diversity of the population will be similar to the conventional DE.

In order to further diversify the populations, we have introduced an information sharing mechanism. This mechanism allows the population of individuals with a record of higher fitness values to share the search information with other population members. Let us consider that the three populations $P_{1}, P_{2}$ and $P_{3}$ are initialized randomly. $P_{1}$ is set to search for thick blood vessel pixels, $P_{2}$ is set to search for thin blood vessel pixels and $P_{3}$ is set to search for non-blood vessel pixels, respectively. This can be considered as a form of opposition based DE (ODE), though ODE uses only one population [Rahnamayan et al., 2008].

Let $P_{1}^{G}, P_{2}^{G}$ and $P_{3}^{G}$ denote the three populations in their $G^{t h}$ generation. Each $P_{k}^{G}$ consists of NP-max and Ddimensional vectors, as follows:

$$
P_{1}^{G}=\left\{\vec{X}_{1, G}^{(k)}, \vec{X}_{2, G}^{(k)} \ldots \vec{X}_{N P, G}^{(k)}\right\}
$$

where $\vec{X}_{j, G}^{(k)}=\left[\vec{X}_{j, 1, G}^{(k)}, \vec{X}_{j, 2, G}^{(k)} \ldots \vec{X}_{j, D, G}^{(k)}\right] \quad \mathrm{j}=1$ to NP and $\mathrm{k}=1$ to 3 .

To compute the positioning of individuals between populations and the $(G+1)^{(t h)}$ generation, we define the following:

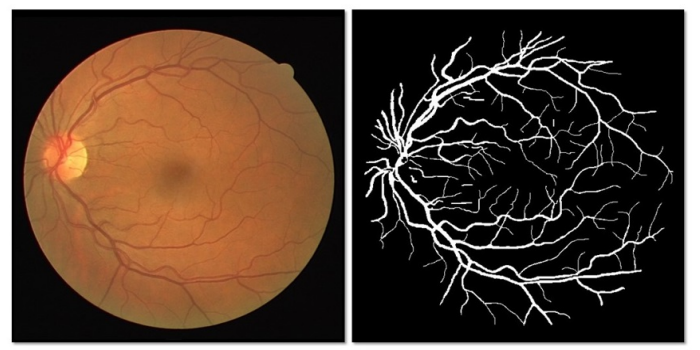

Fig. 3. Segmentation after applying proposed DE to the retinal image

$\mathrm{F}_{j}\left(\left[P_{1}^{G}, P_{2}^{G}\right]\right)=$
$\left\{\begin{array}{l}{\left[P_{1}^{G}, P_{2}^{G}\right] \quad \text { if } \vec{X}_{j, G}^{(1)} \leq \max _{k=1 \text { to5 }} f\left(\vec{X}_{k, G}^{(2)}\right)} \\ \left(P_{1}^{G} \backslash\left\{\vec{X}_{j, G}^{(1)}\right\}\right) \cup\left\{\vec{X}_{\arg }^{(2)} \min _{k=1 t o 5} f\left(\vec{X}_{k, G}^{(2)}\right), G\right\}, \\ P_{2}^{G} \backslash\left\{\vec{X}_{\arg }^{(2)} \min _{k=1 t o 5}, f\left(\vec{X}_{k, G}^{(2)}\right), G\right\} \quad \text { otherwise }\end{array}\right.$

$\left[\mathrm{P}_{1}^{(G+1)}, P_{2}^{(G+1)}\right]=$

$\mathrm{F}_{N P}\left(F_{(N P-1)}\left(F_{(N P-2)}\right) \ldots F_{2}\left(F_{1}\left(\left[P_{1}^{G}, P_{2}^{G}\right]\right)\right)\right)$

The algorithm's ability to perform automatic threshold selection while preserving main features from the original image was observed as in [13]. The important control parameters used in $\mathrm{DE}$ are: population size $\mathrm{N}$, variation factor $\mathrm{F}$ and crossover probability $\mathrm{CR}$ etc. The $\mathrm{DE}$ is set to run 200 iterations over all the populations with different search criteria. After 30 iterations the member of $P_{1}$ will have higher fitness values, because compared to other feature pixels the thick blood vessels pixels are easy to identify and have distinctive features. So, after 30 iterations (selected based on experiments) we will compare each individual from $P_{1}$ with the individuals from $P_{2}$ and $P_{3}$. The population with the highest correlating members will be selected for information sharing. The fitness of individuals from $P_{1}$ will be evaluated using the search criteria used by selected population. If any member of $P_{1}$ is able to achieve higher or similar fitness than the top five members of the selected population, then that individual will be swapped by weakest member of selected population. This mechanism is able to improve the diversity of population and avoids the situation of local stagnation. The fitness function used to evaluate each individual is given as follows:

$$
F(x)=w_{a} * a c c_{x}+w_{f} *(\text { number_feature })^{-1}
$$

where $w_{a}$ and $w_{f}$ are two predefined constant weights for $a c c_{x}$ (classification accuracy) and number_feature (the $_{x}$ number of features), respectively. In this paper, $w_{a}=0.8$ and $w_{f}=0.2$, with $w_{a}>w_{f}$ to represent the fact that classification accuracy is more important than the number of selected features. An example of segmentation after applying proposed DE to the retinal image is shown in figure 3 . The algorithm 1 shows the steps used. 
Algorithm 1: The Pseudo Code of the Modified DE

Initialize three sets of the population.

Evaluate the fitness value for each individual using the

Equation 10, with separate criteria for each

population.

while (satisfying termination criteria) do

Perform standard DE steps as follows:

Mutation using Equation 3.

Crossover using Equation 4 and 5.

Selection using Equation 6.

Re-evaluate the fitness value for each individual in the current population.

if (iterations $>=30$ ) then

Compare population 1 with two other populations.

Evaluate fitness for each individual in population 1 with fitness criteria of the most correlating population.

Swap individuals from population 1 if their fitness is higher than top 5 individuals from selected population as in Equation 8.

end

Re-evaluate the fitness value for each individual in population 1 .

end

End of algorithm

Different classifiers have been used to classify vessels and non-vessel pixels. The selected classifiers are popular in image analysis and have proved to show impressive performance as in [14] and [15]. Literature shows that ensemble classifier enhances the performance of the single classifiers as in [14]. NN, SVM, and the SVM-based and NN-based ensembles with SVM and NN as base classifiers respectively carry out the vessel and non-vessel classification. The input layer for $\mathrm{NN}$ is assigned to a number of features extracted from the proposed model. The NN classifier contains a secret layer along with an output layer with 2 nodes denoting vessel or non-vessel. Furthermore, the grid-search mechanism is availed to generate the most favourable parameter settings for the SVM classifier with the aim of achieving optimal performance. The most favourable settings generated for every single model NN and SVM previously described are also applied to the settings of each base classifier within each ensemble. Both ensembles utilize three base classifiers and a weighted majority voting collaboration method to generate the final categorization. Overall, the NN and SVM-based ensembles achieve the best accuracy when subjected to the images from the databases used as described in the next section. The overall system architecture is as shown in figure 4.

There are other non-retinal image processing that uses DE for image segmentation, where the segmentation process is considered to be an optimization problem. [16] proposed a beta differential evolution (BDE) algorithm for determining the (n-1) optimal n-level threshold on a given image. Cuevas

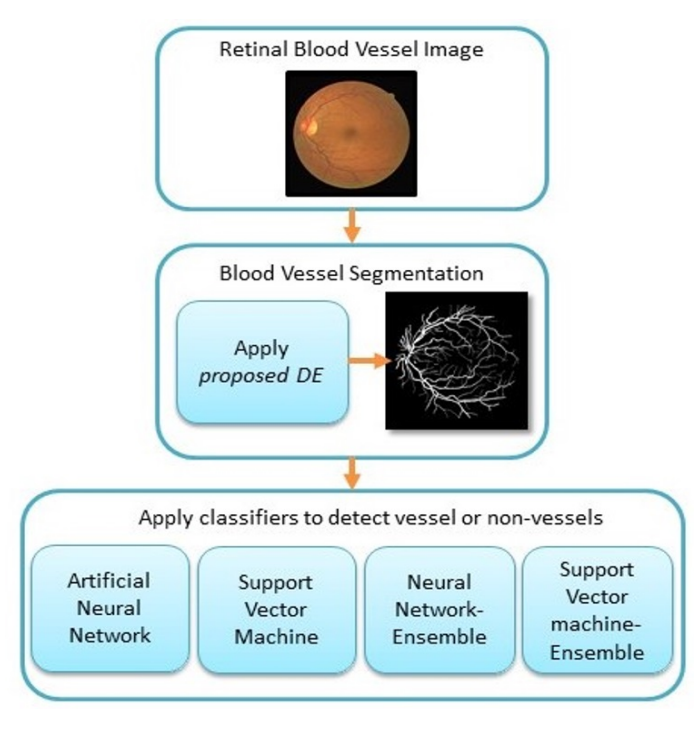

Fig. 4. Main stages of the overall system

et al. [13] used the DE algorithm to approximate the 1D histogram of the image using a mixture of Gaussian functions, representing a threshold point, whose parameters are calculated using the DE method.

\section{Evaluation}

We have proposed an automatic retinal blood vessel segmentation system, which segments blood vessels from fundus image and outperforms most of the existing methodologies. The proposed system is evaluated using three datasets i.e. DRIVE [12], STARE [17], and HRF [18]. The STARE (STructured Analysis of the Retina) Project was conceived and initiated in 1975 by Michael Goldbaum, M.D., at the University of California, San Diego, USA. The STARE database has a set of around 400 raw retinal images. The DRIVE (Digital Retinal Images for Vessel Extraction) dataset was obtained from a diabetic retinopathy screening program in the Netherlands. The screening population consisted of 400 diabetic subjects between 25-90 years of age. Forty photographs have been randomly selected, 33 do not show any sign of diabetic retinopathy and 7 show signs of mild early diabetic retinopathy. The set of 40 images has been divided into a training and a test set, both containing 20 images. The HRF (High-Resolution Fundus) dataset has 15 retinal images of healthy patients, 15 images of patients with diabetic retinopathy and 15 images of glaucomatous patients which is gathered through a collaborative research group in Germany.

The proposed system is implemented from scratch using $\mathrm{C}++$ and OpenCV library under Ubuntu operating system. The learning algorithms such as NN, SVM, NN- and SVMbased ensemble are imported from OpenCV and LibSVM library. The ground truth of the matching image is used to evaluate the performance of the proposed methodology on segmenting vessels from a fundus image. In order to measure the performance of the proposed system, we use accuracy, sensitivity, and specificity value. To calculate the 
accuracy, sensitivity, and specificity we have to consider four measures i.e., true positives, false positives, false negatives and true negatives. The correctly categorized vessel pixels as vessels are denoted as true positive (TP) and correctly categorized non-vessel pixels as non-vessels are denoted as true negative (TN). Whereas, wrongly categorized nonvessels pixels as vessels are denoted as false positive (FP) and wrongly categorized vessels pixels as non-vessels are denoted as false negative (FN). The equations used to calculate accuracy, sensitivity, and specificity value are as follows:

$$
\begin{gathered}
\text { Accuracy }=((T P+T N)) /((T P+T N+F P+F N)) \\
\text { Sensitivity }=T P /((T P+F N)) \\
\text { Specificity }=T N /((T N+F P))
\end{gathered}
$$

The performance of the proposed system using NN classifier on three selected datasets is illustrated in table 1. Table 1 shows the average accuracy, sensitivity and specificity of all the images in DRIVE, STARE, and HRF datasets. Table 2 presents the performance of the proposed system using SVM classifier on the three selected datasets. Meanwhile, Table 3 and 4 presents the performance of the proposed system using NN-based and SVM-based ensemble classifiers on the three selected datasets. The accuracy is the highest when the proposed system is used with SVM-based ensemble classifier as in table 4 . The trial and error run was used for setting the neural network parameters and grid search was used for setting the SVM parameters.

TABLE I

PERFORMANCE OF PROPOSED SYSTEM WITH NN

\begin{tabular}{|l|l|l|l|}
\hline Dataset & Accuracy & Sensitivity & Specificity \\
\hline DRIVE & 95.1 & 84.5 & 97.1 \\
STARE & 95.0 & 82.0 & 96.3 \\
HRF & 96.3 & 85.2 & 98.1 \\
\hline
\end{tabular}

TABLE II

PERFormance OF PROPOSED SYSTEM WITH SVM

\begin{tabular}{|l|l|l|l|}
\hline Dataset & Accuracy & Sensitivity & Specificity \\
\hline DRIVE & 95.9 & 85.8 & 98.0 \\
STARE & 95.2 & 86.8 & 98.1 \\
HRF & 95.8 & 85.0 & 98.6 \\
\hline
\end{tabular}

TABLE III

PeRformance OF PROPOSED SYSTEM WITH ENSEMBLE NN

\begin{tabular}{|l|l|l|l|}
\hline Dataset & Accuracy & Sensitivity & Specificity \\
\hline DRIVE & 96.5 & 86.4 & 98.4 \\
STARE & 96.7 & 83.8 & 98.5 \\
HRF & 98.6 & 87.5 & 99.2 \\
\hline
\end{tabular}

A comparison of the proposed DE based system using SVM-based ensemble classifier with other existing systems is done as in table 5 (refer to next page). The proposed system is able to show better performance with other methodologies when evaluated with three publicly available datasets. Our results show that ensemble SVM is better than single SVM
TABLE IV

PERFORMANCE OF PROPOSED SYSTEM WITH ENSEMBLE SVM

\begin{tabular}{|l|l|l|l|}
\hline Dataset & Accuracy & Sensitivity & Specificity \\
\hline DRIVE & 97.9 & 88.2 & 98.8 \\
STARE & 98.7 & 86.4 & 99.2 \\
HRF & 99.0 & 89.4 & 99.4 \\
\hline
\end{tabular}

and NN variants as per table 1 to 4 . Also, SVM worked slightly better than NN as per table 1 to 4 . These are supported by the results in [28] and [29]. Table 5 shows that the proposed system outperforms specifically the works of Wang et al., Moghimirad et al., GeethaRamani et al., Imani et al., Franklin et al., Cheng et al., and others when evaluated with DRIVE, STARE and HRF datasets. For the DRIVE dataset, the new proposed algorithm had achieved $97.9 \%$ accuracy, for STARE da-taset $98.7 \%$ accuracy and for HRF dataset $99.0 \%$ accuracy, giving an average accuracy across three datasets to be $98.5 \%$, along with $88 \%$ average sensitivity and $99 \%$ aver-age specificity. The above results show that the proposed system can accurately segment the blood vessels from the retinal fundus images. The further analysis of segmented vessels using the proposed system can lead to automatic disease diagnosis like diabetic retinopathy, artery and vein occlusion, hypertensive retinopathy.

\section{CONCLUSION}

In this paper, we have improved the segmentation and classification for efficient retinal image analysis. The new system uses a modified differential evolution (DE) algorithm to carry out automatic retinal blood vessel segmentation. Multiple classifiers are used to identify whether the retinal blood vessel images are healthy or unhealthy (i.e. with the retinal disease). The proposed system is evaluated using multiple publicly available datasets. Upon evaluation, the proposed system can achieve a high average accuracy of $98.5 \%$ on three datasets, which outperforms most of the systems reported in the literature. This work can help in diagnosing the retinal diseases at an earlier stage.

\section{REFERENCES}

[1] World Health Organization (WHO). Universal eye health: A global action plan 2014-2019, WHO Press, Geneva, Switzerland, 2013.

[2] R. GeethaRamani Lakshmi Balasubramanian. Retinal blood vessel segmentation employing image processing and data mining techniques for computerized retinal image analysis, Biocybernetics and Biomedical Engineering, 36(1):102-118, 2016.

[3] R. Storn and K. Price. Journal of Global Optimization 11: 341, 1997.

[4] S. Das and P. N. Suganthan, "Differential Evolution: A Survey of the State-of-the-Art," in IEEE Transactions on Evolutionary Computation, vol. 15, no. 1, pp. 4-31, 2011.

[5] S. Wilfred Franklin and S. Edward Rajan. Computerized screening of diabetic retinopathy employing blood vessel segmentation in retinal images. Biocybern Biomed Eng 34:117-24, 2014.

[6] E. Emary, H. M. Zawbaa, A. E. Hassanien, G. Schaefer and A. T. Azar, "Retinal blood vessel segmentation using bee colony optimisation and pattern search," 2014 International Joint Conference on Neural Networks (IJCNN), Beijing, pp. 1001-1006, 2014.

[7] G. Hassan, A. E. Hassanien, N. El-bendary and A. Fahmy, "Blood vessel segmentation approach for extracting the vasculature on retinal fundus images using Particle Swarm Optimization," 2015 11th International Computer Engineering Conference (ICENCO), Cairo, pp. 290-296, 2015. 
TABLE V

PERFORMANCE COMPARISON OF THE PROPOSED WORK WITH STATE-OF-THE-ART

\begin{tabular}{|l|l|l|l|l|l|}
\hline Dataset & Authors and Year & Accuracy & Sensitivity & Specificity & Algorithm/Approach \\
\hline DRIVE & *Proposed work, 2018 & 97.9 & 88.2 & 98.8 & *New DE \& Ens-SVM \\
DRIVE & Wang et al., 2015 [11] & 97.7 & 81.7 & 97.3 & CNN and RF \\
DRIVE & Moghimirad et al., 2012 [19] & 96.6 & 78.5 & 99.4 & Multi-scale method \\
DRIVE & GeethaRamani et al., 2016 [2] & 95.4 & 70.8 & 97.8 & DT with Bagging \\
DRIVE & Imani et al., 2015 [20] & 95.2 & 75.2 & 97.5 & MCA approach \\
DRIVE & Franklin and Rajan, 2014 [5] & 95.0 & 68.7 & 98.2 & MP Neural Network \\
DRIVE & Roychowdhury et al., 2015 [21] & 95.2 & 72.5 & 98.3 & GMM classifier \\
DRIVE & Strisciuglio et al, 2015 [22] & 94.7 & 77.3 & 97.2 & SVM with GMLVQ \\
DRIVE & Liu et al., 2014 [23] & 94.7 & 73.5 & 97.7 & Multiple Kernel SVM \\
\hline STARE & *Proposed work, 2018 & 98.7 & 86.4 & 99.2 & *New DE \& Ens-SVM \\
STARE & Wang et al., 2015 [11] & 98.1 & 81.0 & 97.9 & CNN and RF \\
STARE & Moghimirad et al., 2012 [19] & 97.6 & 81.3 & 99.1 & Multi-scale method \\
STARE & Imani et al., 2015 [20] & 95.9 & 75.0 & 97.5 & MCA approach \\
STARE & Annunziata, et al., 2015 [24] & 95.6 & 71.3 & 98.4 & NEBF approach \\
STARE & Roychowdhury et al., 2015 [21] & 95.2 & 77.2 & 97.3 & GMM classifier \\
STARE & Strisciuglio et al, 2015 [22] & 95.4 & 80.1 & 97.2 & SVM with GMLVQ \\
STARE & Liu et al., 2014 [23] & 95.7 & 76.3 & 97.1 & Multiple Kernel SVM \\
\hline HRF & *Proposed work, 2018 & 99.0 & 89.4 & 99.4 & *New DE \& Ens-SVM \\
HRF & Cheng et al., 2014 [25] & 96.1 & 70.4 & 98.6 & RF with SWT \& WLD \\
HRF & Christodoulidis et al, 2016 [26] & 94.8 & 85.1 & 95.8 & Multi-scale Voting \\
HRF & Annunziata, et al., 2015 [24] & 95.8 & 71.3 & 98.4 & NEBF approach \\
HRF & Lázár et al., 2015 [27] & 95.3 & 71.0 & 98.3 & Direc. Resp. Vector \\
HRF & Odstrcilik et al., 2013 [18] & 94.9 & 77.4 & 96.7 & Impr. Matched Filter \\
\hline
\end{tabular}

[8] S. Kim, S. Nowozin, P. Kohli, C. D. Yoo, Higher-Order Correlation Clustering for Image Segmentation, Proceedings of Neural Information Processing Systems (NIPS 2011), Advances in Neural Information Processing Systems 24, pp.1-9.

[9] K. S. Sreejini, V. K. and Govindan, Improved multiscale matched filter for retinal vessel segmentation using PSO algorithm, Egyptian Informatics Journal, 16(3), pp.253-260, 2015.

[10] R. Arnay, F. Fumero and J. Sigut, Ant Colony Optimization-based method for optic cup segmentation in retinal images, Applied Soft Computing, 52, pp. 409-417, 2017.

[11] S. Wang, Y. Yin, G. Cao, B. Wei, Y. Zheng, and G. Yang, Hierarchical retinal blood vessel segmentation based on feature and ensemble learning, Neurocomputing, 149(B), pp. 708-71, 2015.

[12] J. Staal, M. D. Abramoff, M. Niemeijer, M. A. Viergever and B. van Ginneken. Ridge-based vessel segmenta-tion in color images of the retina. IEEE Trans Med Imaging 23(4):501-9, 2004.

[13] E. Cuevas et al., Applications of Evolutionary Computation in Image Processing and Pattern Recognition, Intelligent Systems Reference Library 100, 2016.

[14] K. Mistry, L. Zhang, S. C. Neoh, C. P. Lim, and B. Fielding. A micro-GA Embedded PSO Feature Selection Approach to Intelligent Facial Emotion Recognition. IEEE Transactions on Cybernetics, 47(6), pp.1496 - 1509, 2016.

[15] S. C. Neoh, L. Zhang, K. Mistry, M. A. Hossain, C. P. Lim, N. Aslam, and P. Kinghorn. "Intelligent Facial Emotion Recognition Using a Layered Encoding Cascade Optimization Model”. Appl Soft Comput. Volume 34, 72-93, 2015.

[16] H. V. H. Ayala, F. M. D. Santos, V. C. Mariani and L. Coelho. Image thresholding segmentation based on a novel beta differential evolution approach, Expert Systems with Applications, 42(4), 2014.

[17] A. D. Hoover, V. Kouznetsova and M. Goldbaum. Locating blood vessels in retinal images by piecewise threshold probing of a matched filter response. IEEE Trans. Med. Imaging 19, 203-210, 2000.

[18] J. Odstrcilik, R. Kolar, A. Budai, J. Hornegger, J. Jan, J. Gazarek, T. Kubena, P. Cernosek, O. Svoboda and E. Angelopoulou. Retinal vessel segmentation by improved matched filtering: evaluation on a new high-resolution fundus image database. IET Image Process. 7, 373-383, 2013.

[19] E. Moghimirad, S. Hamid Rezatofighi, and H. Soltanian-Zadeh. Retinal vessel segmentation using a multi-scale medialness function. Comput. Biol. Med. 42:50-60, 2012.

[20] E. Imani, M. Javidi, and H-R Pourreza. Improvement of retinal blood vessel detection using morphological component analysis. Comput. Methods Progr. Biomed. 118:263-279, 2015.

[21] S. Roychowdhury, D.D. Koozekanani, and K. K. Parhi. Blood vessel segmentation of fundus images by major vessel extraction and subimage classification. IEEE J. Bi-omed. Health Inf. 19, 1118-1128, 2015.

[22] N. Strisciuglio, G. Azzopardi, M. Vento and N. Petkov. Multiscale blood vessel delineation using B-cosfire filters. In: International Conference on Computer Analysis of Images and Patterns. Springer, pp. 300-312, 2015.

[23] X. Liu, Z. Zeng and X. Wang. Vessel segmentation in retinal images with a multiple kernel learning based method. In: 2014 International Joint Conference on Neural Networks (IJCNN). IEEE, pp. 507-511, 2014.

[24] R. Annunziata, A. Garzelli, L. Ballerini, A. Mecocci, and E. Trucco. Leveraging Multiscale Hessian-Based Enhancement with a Novel Exudate Inpainting Technique for Retinal Vessel Seg-mentation. IEEE Journal Biomed Health Inform. 20(4):1129-38, 2015.

[25] E. Cheng, L. Du, Y. Wu, Y. J. Zhu, V. Megalooikonomou, and H. Ling. Discriminative vessel segmentation in retinal images by fusing context-aware hybrid features. Mach. Vis. Appl. 25:1779-1792, 2014.

[26] A. Christodoulidis, Thomas Hurtut, Houssem Ben Tahar, and Farida Cheriet. A multi-scale tensor voting approach for small retinal vessel segmentation in high-resolution fundus images. Comput. Med. Imaging Graph. 52:28-43, 2016.

[27] I. Lázár and A. Hajdu. Segmentation of retinal vessels by means of directional response vector similarity and region growing. Comput. Biol. Med. 66, 209-221, 2015.

[28] H. Kim, S. Pang, H. Je, D. Kim, and S. Y. Bang, Constructing support vector machine ensemble, Pattern Recognition, Volume 36, Issue 12, 2003.

[29] D. S. Jodas, N. Marranghello, A. S. Pereira, R. C. Guido, Comparing Support Vector Machines and Artificial Neural Networks in the Recognition of Steering Angle for Driving of Mobile Robots Through Paths in Plantations, Proceedings of the International Conference on Computational Science (ICCS 2013), Procedia Computer Science, Volume 18, 2013, 240-249. 